\section{VALIDATION OF PD-L1 DYNAMIC EXPRESSION ON EXTRACELLULAR VESICLES AS A PREDICTOR OF RESPONSE TO IMMUNE-CHECKPOINT INHIBITORS AND SURVIVAL IN NON-SMALL CELL LUNG CANCER PATIENTS}

\begin{abstract}
${ }^{1}$ Diego de Miguel Perez ${ }^{*},{ }^{2}$ Feliciano Barrón, ${ }^{3}$ Alessandro Russo, ${ }^{2}$ Luis Lara-Mejía, ${ }^{4}$ Muthukumar Gunasekaran, ${ }^{5}$ Andrés Cardona, ${ }^{6}$ Christine Peterson, ${ }^{7}$ Rivka Colen, ${ }^{6}$ Aung Naing, ${ }^{8}$ Philip Mack, ${ }^{8}$ Fred Hirsch, ${ }^{9}$ Vincenzo Adamo, ${ }^{2}$ Oscar Arrieta, ${ }^{1}$ Christian Rolfo. ${ }^{1}$ Tisch Cancer Institute and Icahn School of Medicine, Mount Sinai, NY and Marlene and Stewart Greenebaum Comprehensive Cancer Center, University of Maryland School of Medicine, New York, NY, USA; ${ }^{2}$ Instituto Nacional de Cancerología (INCAN), Mexico city, Mexico; ${ }^{3}$ A.O. Papardo and Department of Human Pathology, University of Messina and Marlene and Stewart Greenebaum Comprehensive Cancer Center, University of Maryland School of Medicine, Messina, Italy; ${ }^{4}$ University of Maryland School of Medicine, Baltimore, MD, USA; ${ }^{5}$ Clínica del Country, Bogota, Colombia; ${ }^{6}$ The University of Texas MD Anderson Cancer Center, Houston, TX, USA; ${ }^{7}$ University of Pittsburgh, Pittsburgh, PA, USA; ${ }^{8}$ Tisch Cancer Institute and Icahn School of Medicine, Mount Sinai, NY, New York, NY, USA; ${ }^{9}$ A. O. Papardo and Department of Human Pathology, University of Messina, Messina, Italy
\end{abstract}

Background Immune-checkpoint inhibitors (ICIs) revolutionized the treatment of advanced non-small cell lung cancer (NSCLC). ${ }^{1-3}$ To date, tissue PD-L1 immunohistochemistry is one of the leading biomarkers for prediction of ICIs response but has several limitations. ${ }^{4}{ }^{5}$ Extracellular vesicles (EVs) are cell-derived structures involved in cell communication and represent a potential minimally invasive alternative to predicting ICI response. ${ }^{6-9}$ Based on this and our preliminary results presented at SITC $2020,{ }^{10}$ we hypothesize that EV PD-L1 predicts response to ICIs in NSCLC.

Methods This study evaluates an exploratory cohort of advanced/metastatic NSCLC patients receiving ICIs (cohort A) and a validation cohort receiving Pembrolizumab+docetaxel or docetaxel alone (PROLUNG Phase 2 randomized trial) (cohort B). ${ }^{11}$ Plasma samples were collected pre-treatment (T1) and at 3 treatment cycles (T2) (figure 1A). Response was assessed by computed-tomography scan at 3 (cohort A) and 6-8 treatment cycles (cohort B) according to mono- or chemotherapy combination therapy. Patients were classified as responders (partial, stable, or complete response) or non-responders (progressive disease) by RECISTv1.1. ${ }^{12}$ EVs were isolated by serial ultracentrifugation and characterized following ISEV recommendations. ${ }^{13,14}$ Tissue PD-L1 expression was measured by standardized immunohistochemistry (SP263, 22C3, or 28-8 clones) ${ }^{5}$ and EV PD-L1 expression by immunoblot and its ratio was calculated as EV PD-L1 T2/T1. Cut-offs from the exploratory cohort were applied to the validation cohort, being EV PD-L1 ratio $<0.85=$ Low.

Results Paired samples from 30 ICIs, 23 pembrolizumab+doceetaxel, and 15 docetaxel treated patients were analyzed. In cohort A, non-responders showed higher EV PD-L1 ratio than responders $(\mathrm{p}=0.012)$ (figure $1 \mathrm{~B})$ with an area-under-the-curve (AUC) of $77.3 \%, 83.3 \%$ sensitivity, and $61.1 \%$ specificity, while the tissue PD-L1 was not predictive (AUC=50\%). As a validation, pembrolizumab+docetaxel treated non-responders showed higher EV PD-L1 ratio $(\mathrm{p}=0.036)$ than responders with an $\mathrm{AUC}=69.3 \%$, sensitivity $=75 \%$, and specificity $=63.6 \%$, outperforming the tissue PD-L1 (figure 1C). No statistically significant differences were observed in the docetaxel group $(p=0.885)$. Moreover, ICIs patients with higher EV PD-L1 ratio showed shorter progression-free survival (PFS) $(\mathrm{HR}=0.30, \mathrm{p}=0.066)$ and overall survival (OS) $(\mathrm{HR}=0.17$, $\mathrm{p}=0.016$ ) (figure $1 \mathrm{D}$ ) which was also observed in the pembrolizumab+docetaxel cohort with shorter PFS $(\mathrm{HR}=0.12$, $\mathrm{p}=0.004)$ and $\mathrm{OS}(\mathrm{HR}=0.23, \mathrm{p}=0.010)$ (figure $1 \mathrm{E})$. EV PDL1 ratio did not predict survival in docetaxel-treated patients.

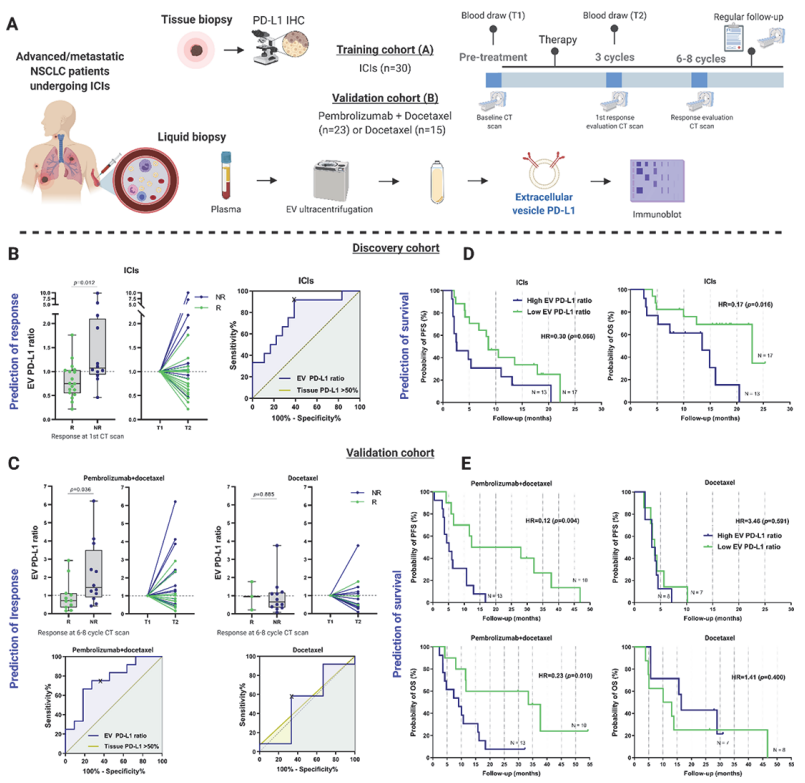

Abstract 23 Figure 1 (A) Study design and methodology. (B) EV PD$\mathrm{L} 1$ ratio predicts response to ICls in $30 \mathrm{NSCLC}$ patients from the discovery cohort A and outperforms tissue PD-L1. (C) EV PD-L1 ratio is predictive for response to pembrolizumab+docetaxel in 23 NSCLC patients but not in 15 patients receiving docetaxel alone from cohort $B$. (D) Higher EV PD-L1 ratio predicts shorter PFS and OS in 30 patients from the discovery cohort A treated with ICls. (E) Higher EV PD-L1 ratio is associated with shorter PFS and OS in 23 patients treated with pembrolizumab+docetaxel but not in patients treated with docetaxel alone. Abbreviations: CT: Computed tomography, EV: Extracellular vesicle; HR: Hazard Ratio; ICls: Immune-checkpoint Inhibitors; IHC: Immunohistochemistry; NR: Non-Responders; OS: Overall Survival; p: pvalue; PFS: Progression-free survival; R: Responders [Created with BioRender].

Conclusions We demonstrated that treatment-associated changes in EV PD-L1 levels are predictive of response and survival in advanced NSCLC patients treated with ICIs. This model, if confirmed in a large prospective cohort, could have important clinical implications, guiding treatment decisions and improving the outcome of patients receiving ICIs.

Acknowledgements We would like to extend our gratitude to the all the patients that participated in the study.

\section{REFERENCES}

1. Borghaei H, Paz-Ares L, Horn L, Spigel DR, Steins M, Ready NE, et al. Nivolumab versus Docetaxel in Advanced Nonsquamous Non-Small-Cell Lung Cancer. N Engl J Med 2015;373:1627-39

2. Herbst RS, Baas P, Kim DW, Felip E, Pérez-Gracia JL, Han JY, et al. Pembrolizumab versus docetaxel for previously treated, PD-L1-positive, advanced non-smallcell lung cancer (KEYNOTE-010): A randomised controlled trial. Lancet 2016:387:1540-50.

3. Ruiz-Patiño A, Arrieta O, Cardona AF, Martín C, Raez LE, Zatarain-Barrón ZL, et al. Immunotherapy at any line of treatment improves survival in patients with advanced metastatic non-small cell lung cancer (NSCLC) compared with chemotherapy (Quijote-CLICaP). Thorac Cancer 2020;11:353-61.

4. Doroshow DB, Bhalla S, Beasley MB, Sholl LM, Kerr KM, Gnjatic S, et al. PD-L1 as a biomarker of response to immune-checkpoint inhibitors. Nat Rev Clin Oncol 2021;18:345-362.

5. Hirsch FR, McElhinny A, Stanforth D, Ranger-Moore J, Jansson M, Kulangara K, et al. PD-L1 immunohistochemistry assays for lung cancer: results from phase 1 
of the blueprint PD-L1 IHC assay comparison project. I Thorac Oncol 2017;12:208-222.

6. Poggio M, Hu T, Pai CC, Chu B, Belair CD, Chang A, et al. Suppression of exosomal PD-L1 induces systemic anti-tumor immunity and memory. Cell 2019;177:414-427.e13.

7. Cordonnier M, Nardin C, Chanteloup G, Derangere V, Algros MP, Arnould L, et al. Tracking the evolution of circulating exosomal-PD-L1 to monitor melanoma patients. J Extracell Vesicles 2020;9:1710899.

8. Del Re M, Cucchiara F, Rofi E, Fontanelli L, Petrini I, Gri N, et al. A multiparametric approach to improve the prediction of response to immunotherapy in patients with metastatic NSCLC. Cancer Immunol Immunother 2020;70:16671678.

9. Chen $G$, Huang AC, Zhang W, Zhang G, Wu M, Xu W, et al. Exosomal PD-L1 contributes to immunosuppression and is associated with anti-PD-1 response. Nature. 2018;560:382-6.

10. 10 de Miguel Perez D, Russo A, Gunasekaran M, Cardona A, Lapidus R, Cooper $B$, et al. 31 Dynamic change of PD-L1 expression on extracellular vesicles predicts response to immune-checkpoint inhibitors in non-small cell lung cancer patients. 2020J Immunother Cancer;8(Suppl 3):A30-A30.

11. Arrieta $\mathrm{O}$, Barrón $\mathrm{F}$, Ramírez-Tirado LA, Zatarain-Barrón ZL, Cardona AF, DíazGarcía D, et al. Efficacy and safety of pembrolizumab plus docetaxel vs docetaxel alone in patients with previously treated advanced non-small cell lung cancer: the PROLUNG phase 2 randomized clinical trial. 2020JAMA Oncol;6:856-864.

12. Eisenhauer EA, Therasse P, Bogaerts J, Schwartz LH, Sargent D, Ford R, et al. New response evaluation criteria in solid tumours: Revised RECIST guideline (version 1.1). 2009Eur I Cancer;45:228-47.

13. Reclusa P, Verstraelen P, Taverna S, Gunasekaran M, Pucci M, Pintelon I, et al. Improving extracellular vesicles visualization: From static to motion. 2020Sci Rep; $10: 6494$

14. Théry C, Witwer KW, Aikawa E, Alcaraz MJ, Anderson JD, Andriantsitohaina R, et al. Minimal information for studies of extracellular vesicles 2018 (MISEV2018): a position statement of the International Society for Extracellular Vesicles and update of the MISEV2014 guidelines. 2018J Extracell Vesicles;7:1535750

Ethics Approval Patients consented to Institutional Review Board-approved protocol, A.O. Pappardo, Messina, Italy for cohort A and Thoracic Oncology Unit, Instituto Nacional de Cancerología (INCan), México City, México in case of the cohort B. Biological material was transferred to the University of Maryland School of Medicine, Baltimore for EV analysis under signed MTA between institutions MTA/2020-13111 \& MTA/2020-13113.

http://dx.doi.org/10.1136/jitc-2021-SITC2021.023 